\title{
Sunflower Optimization Algorithm Based Filtering Method for State of Charge Estimation of Batteries in Electric Vehicle
}

\section{A Maheshwari ( $\sim$ maheshwariphd19@gmail.com )}

Alagappa Chettiar Government College of Engineering and Technology https://orcid.org/0000-00027721-8679

\section{S. Nageswari}

Alagappa Chettiar Government College of Engineering and Technology

\section{Research Article}

Keywords: Battery model, Battery Management System, Electric Vehicle, Kalman filter, Li-ion battery, State Estimation.

Posted Date: November 9th, 2021

DOI: https://doi.org/10.21203/rs.3.rs-1024909/v1

License: (c) (i) This work is licensed under a Creative Commons Attribution 4.0 International License. Read Full License 


\title{
Sunflower Optimization Algorithm Based Filtering Method for State of Charge Estimation of Batteries in Electric Vehicle
}

\author{
A. Maheshwari ${ }^{1}$, S. Nageswari ${ }^{2}$ \\ ${ }^{1,2}$ Department of Electrical and Electronics Engineering \\ ${ }^{1,2}$ Alagappa Chettiar Government College of Engineering and Technology, Karaikudi. \\ ${ }^{1}$ maheshwariphd19@gmail.com, ${ }^{2}$ nageswaribashyam@gmail.com
}

Abstract

The main focus of the battery management system is the estimation of the battery's State of Charge (SOC) which is an indicator to determine the driving range of an electric vehicle. The Extended Kalman Filter (EKF) algorithm is the most promising for SOC estimation when the system is running. The EKF state estimation algorithm is sensitive to the process noise covariance matrix $\mathrm{Q}$ and measurement noise covariance matrix R. Inappropriate noise covariance matrices reduce the accuracy and make divergence in state estimation. In this paper, the Sunflower Optimization algorithm (SFO) is used to optimize the noise covariance matrices before applying EKF for online SOC estimation. This simply indicates that the iterative SFO does not affect the instantaneous response of EKF in online estimation because the SFO is only performed once to determine the optimal values. The effectiveness of the proposed identification is examined through the constant discharge rate test and dynamic stress test. As observed, the performance indices such as maximum error, Mean Absolute Error, Mean Square Error and Root Mean Square Error of both SOC and voltage obtained by the proposed SFO-EKF are low compared to the other three methods. Besides accuracy, the proposed method quickly converges even when the initial SOC is inaccurate. The simulation results show that the proposed method has high accuracy and a better convergence rate in terms of estimating SOC under static and dynamic operating conditions.

Keywords: Battery model, Battery Management System, Electric Vehicle, Kalman filter, Li-ion battery, State Estimation.

\section{INTRODUCTION}

Electric Vehicles (EVs) play a key role in solving the environmental problems in the world. Due to factors such as climate change, advances in renewable energy, battery chemistry, 
rapid urbanization, data capture \& analysis and energy security, most developed and developing countries have included EVs in their policies to mitigate carbon emissions and provide costeffective zero emission vehicles [1]. The rapid development of EV technology has led to research into battery technology, as the battery is an essential component for EVs [2]. Among several batteries, the Li-ion battery is the most suitable one due to its features like high energy density, long life cycle, low self-discharge rate, etc. [3]. Li-ion batteries are dangerous when used outside of their safe operating range, especially when employed in electric vehicles. Because of the harsh operating conditions of EVs, the most prominent battery technology for EVs necessitates a Battery Management System (BMS) for precise monitoring, cell balancing, limiting thermal runaway, and ensuring the battery's life duration [4]. In numerous key functions of BMS, SOC is an indicator to control the charging and discharging limits for the safe use of batteries. SOC, like an energy gauge, measures the amount of charge available in a battery to reduce the range anxiety of the driver [5]. Therefore, precise estimation of SOC is most important for EV. But it is a challenging task since it cannot be directly assessed using any kind of sensor or measuring instrument [6]. It needs to be estimated using measured variables such as current, voltage, and temperature [7], [8]. A large number of SOC estimation methods are available in the literature. SOC can be classified into two categories, such as direct estimation and indirect estimation, popularly known as model-based estimation [9]. The Ampere hour (Ah) integral method and Open Circuit Voltage (OCV) method are direct state estimation methods. In an Ah method, charging and discharging current are integrated over time to find SOC [10]. Although this method is simple to implement, the inaccurate unknown initial SOC and sensor error make it insufficient for EVs [11]. The one-to-one relationship between OCV and SOC is used in the OCV method [12]. Though it is simple and easy to implement, it needs a long rest 
time, so it is not suitable for EVs [13]. However, different estimation methods are used, modelbased estimation methods are now acknowledged as the technology of choice [14]. As a result, the first step is to create a battery model, and model accuracy has a direct impact on state estimation [15]. But, the complex nature of the battery's electrochemical process creates a barrier to battery modeling [16]. Three popular models are the electrochemical model, the black-box model, an equivalent circuit model [16]. The electrochemical model illustrates battery behavior by the concentration of electrolytes, the size of the anode and cathode and the electrochemical process inside the battery. Although this model is very accurate and highly interpretable, it is only suitable for offline design applications [18]. Because of a greater number of parameters that are seldom identifiable and complex electrochemical processes, the computational complexity in the implementation of this model is very high [19]. Black-box models are used to approximate nonlinear mapping between the input and output of a battery from existing samples [7]. Blackbox models are built by artificial neural networks, support vector machines, gaussian process regression, recurrent neural networks, deep neural networks and so on. Without the knowledge of battery chemistry, this model requires a lot of training data to illustrate the battery behavior. Although this model is suitable for Li-ion batteries, it necessitates heavy computation and long training time. Moreover, careful attention is required for the selection of hyperparameters and development of a suitable training algorithm [20]. However, this model not only needs high computation but also requires model accuracy, which depends on the quality and quantity of training data [21], [22]. So, it reduces efficiency in the mapping learning process. Thus, a highspeed mapping learning algorithm is desired to process a large amount of training data [23].

According to the most important modeling requirements in terms of accuracy, configuration effort, computational complexity, and ease of implementation [17], the equivalent 
circuit model (ECM) is the preferred choice for online applications. This model has a concise structure and it describes the dynamic behavior of the battery [7]. The two RC network based ECM is the popular model for Li-ion batteries among the various equivalent circuit models such as the Rint model, the Partnership for a New Generation of Vehicles (PNGV) model, combined model and the RC (resistor-capacitor) network based model [24]. Since the model parameters are time-variant, the Variable Forgetting Factor Recursive Least Square algorithm (VFFRLS) proposed in [25] is used to identify the model parameters.

The Kalman Filter (KF) acts as an optimal estimator in linear systems. The extended version of the Kalman filter, i.e., the EKF algorithm, manipulates the probability and statistics, which are the best ways to estimate the states of a nonlinear system [26]. EKF can estimate the states of time-varying and nonlinear model of battery [27]. EKF requires a battery model, state function, measurement function, real-time battery output data, and associated noise terms. EKF has the advantages of a self-correcting nature and the ability to handle the stochastic uncertainties of a system [28]. On the other hand, the filter performance in the estimation process is greatly influenced by process noise covariance matrix $(\mathrm{Q})$ and measurement noise covariance matrix (R) values. In most cases, these covariance matrices are considered as tuning parameters which are tuned by trial \& error method. However, it is a long-running process and does not guarantee convergence.

To avoid this, the Adaptive Extended Kalman Filter (AEKF) [29], [30] and Adaptive Unscented Kalman Filter (AUKF) [31]-[35] are executed to reduce the estimation error caused by inaccurate noise statistics. It is crucial to set an acceptable size for the moving window, just as it is for the update window. With estimating accuracy and calculation complexity in mind, the size of the moving window $M$ is adjusted through optimization [36]. Therefore, AEKF and 
AUKF improve accuracy with increasing computational complexity [37]. To avoid the computational complexity, a method is required that must balance precision and simplicity to estimate the SOC. According to [38], the filter tuning also significant for the estimation performance in addition to the selection of battery model and filter type. Moreover, these covariance matrices are fixed parameters as per Kalman theory. Instead of determining these parameters in every cycle, an optimization algorithm like the genetic algorithm is used to tune the parameters [39]-[42]. In this paper, a new nature inspired metaheuristic algorithm named the Sunflower Optimization Algorithm (SFO) is introduced to optimize the noise covariance matrices. Recall that, the time-consuming iterative process does not affect the quick response of EKF on state estimation as it is applied only once. After completing the tuning process, the optimized matrices are given as constant parameters to the filter for SOC estimation of an electric vehicle battery.

The main contributions of this paper are,

- A closed-loop model-based SOC estimation algorithm is developed.

- The impact of noise covariance matrices on state estimation using EKF is analyzed.

- The optimal values of the noise covariance matrices are selected by the SFO algorithm.

- An optimized EKF based SOC estimation is executed for different operating conditions of the battery.

Instead of doing experiments in the laboratory, the manufacturer's data sheet and online dataset from the National Aeronautics and Space Administration (NASA) are used for the validation [43]-[47]. Therefore, the time required for testing a battery is saved.

Optimization results are obtained through MATLAB software in offline mode and the obtained results are inserted into the EKF algorithm for the SOC estimation process in online 
mode. The results obtained from simulation show the competition of proposed SFO-EKF with AEKF, GA-EKF and EKF designed with the trial \& error method.

\section{PROPOSED BATTERY EQUIVALENT CIRCUIT MODEL-BASED SOC ESTIMATION}

Despite the assurance of the vehicle's driving range, SOC estimation aids in the calculation of energy and power. Therefore, an accurate SOC is needed for the vehicle in motion, which is not an easy task. A powerful estimation method is needed to estimate SOC that cannot be measured directly using any type of sensors or instruments. A simple battery model with reasonable accuracy is needed for the model-based SOC estimation method.

Equivalent circuit models describe the input-output dynamics of a battery cell. For the ECM based SOC estimation approach, state-space equations are derived from the model and an appropriate algorithm based on control system theory is developed to estimate the internal state of a system. The general methodology is outlined in Figure 1. Input current which might be constant or pulsed is applied to the battery cell. Cell terminal voltage and current are measured, which comprise sensor noise and process noise, respectively. The measured current is injected into the battery model to predict the terminal voltage as well as the state of a system. Measurable value and model value are compared to find the error, which indicates how much the model value matches the real value. Different kinds of feedback methods, e.g., adaptive filter or observer, are employed to adjust the model state, which updates the parameter values of the battery model to match the real battery. The output of the model-based estimator is SOC whose accuracy depends on the battery model and estimation algorithm. 


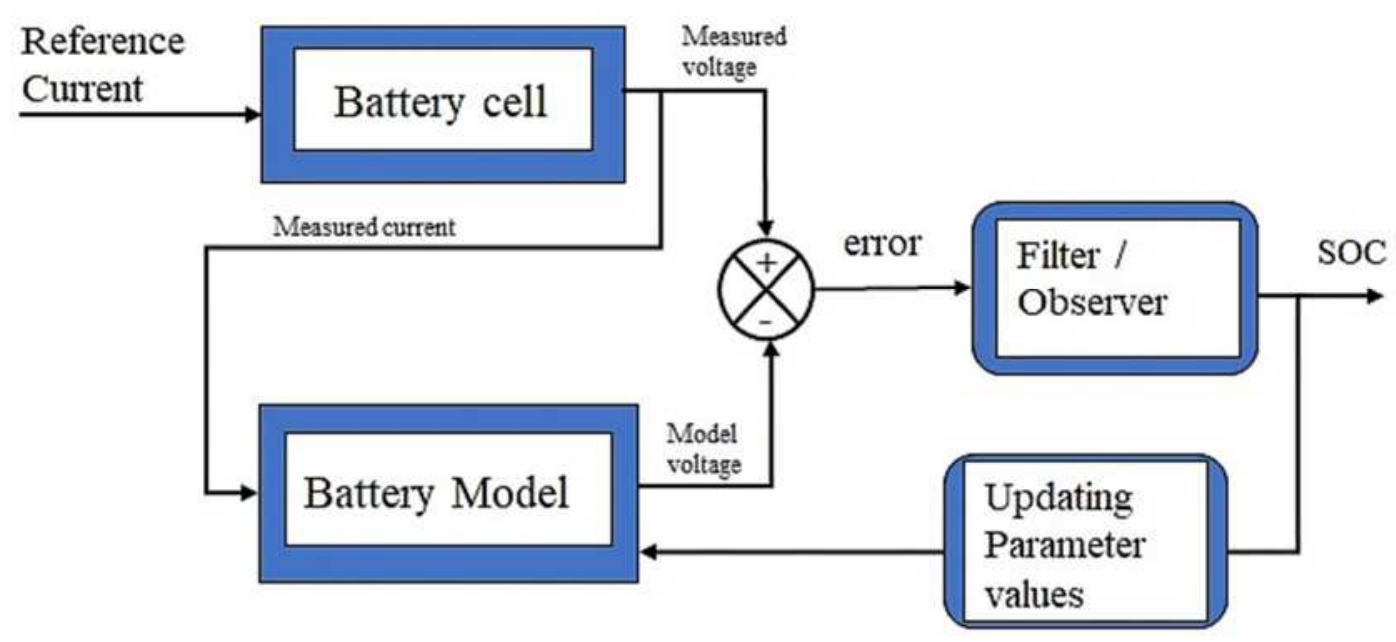

Figure 1 ECM based SOC estimation

From the perspective of control theory, the Filter/Observer in Figure 1 could be considered as a controller. The objective of such a controller is to closely match the battery model with real battery data and finally force the states of the battery model to converge to the states of the real battery.

\subsection{Mathematical Model of a battery:}

Two RC (resistor-capacitor)-ECM is a proven model for SOC estimation due to the best trade-off between accuracy and complexity in real time implementation [48]. Since the model parameters are multivariate functions of temperature, SOC, current, and aging; frequent reparameterization is required. The Recursive Least Square algorithm is a widely used parameter identification method because it gives accurate results with moderate computational effort. The term forgetting factor affects the convergence rate, stability and tracking performance of an algorithm. When the forgetting factor is increased, the convergence rate and stability improve, but the tracking performance suffers [49], [50]. In order to compromise some performance 
criteria such as good stability, fast convergence and improvement in tracking ability, the VFFRLS algorithm is a proven method for battery equivalent circuit model parameter identification [25], [51]. In order to get the relationship between OCV and SOC, the curve fitting tool in MATLAB is used to obtain the fitted curve of OCV and SOC [52].

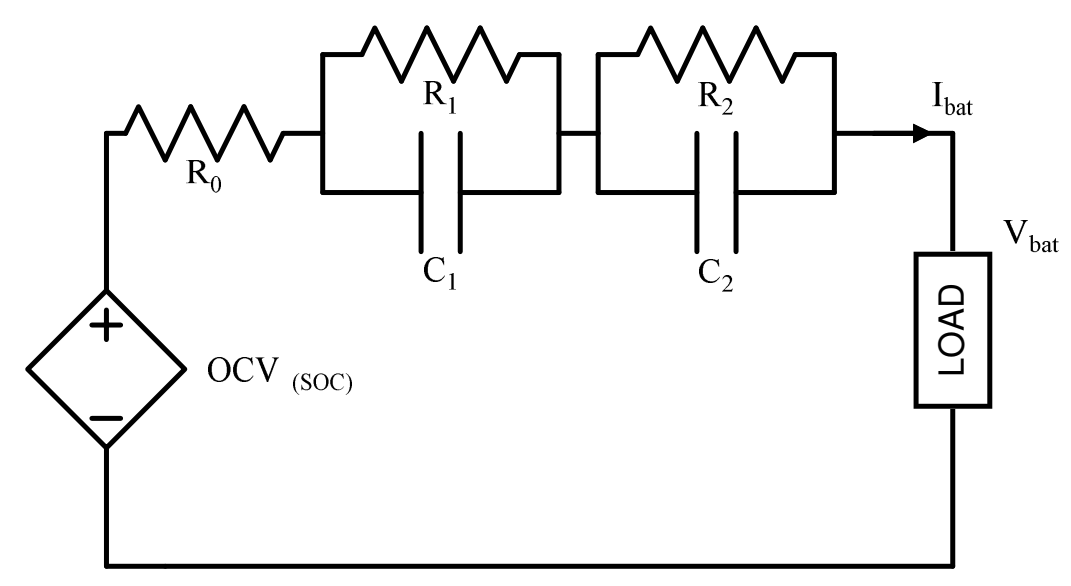

Figure 2 Circuit diagram of Two RC-ECM

Figure 2 shows the schematic diagram of the battery equivalent circuit model which comprises a controlled voltage source and two RC parallel networks with series resistance. The polarization characteristics of the battery are represented by two RC parallel networks in the model. The activation polarization is described by one, while the concentration polarization is described by the other. $V_{1}$ and $V_{2}$ are the transient voltages of the two RC parallel networks, respectively. According to circuit theory, the equations for Two RC-ECM for discharging scenarios under constant current are given by (1-6).

$$
\begin{aligned}
& V_{b a t}=O C V_{(S O C)}-I_{b a t} * R_{0}-V_{1}-V_{2} \\
& I_{b a t}=\frac{V_{1}}{R_{1}}+C_{1} \frac{d V_{1}}{d t}=\frac{V_{2}}{R_{2}}+C_{2} \frac{d V_{2}}{d t}
\end{aligned}
$$


$V_{1}=\left(\frac{Q_{u}}{C_{1}}+I_{b a t} * R_{1}\right) * \exp \left(\frac{-t}{R_{1} * C_{1}}\right)-I_{b a t} * R_{0}$

$V_{2}=\left(\frac{Q_{u}}{C_{2}}+I_{b a t} * R_{2}\right) * \exp \left(\frac{-t}{R_{2} * C_{2}}\right)-I_{b a t} * R_{0}$

where,

$V_{b a t}$ - Output voltage of a battery

$I_{b a t}-$ Input current of a battery

$V_{1} \& V_{2}-$ Voltages across $\mathrm{RC}$ networks

$O C V$ - Open circuit voltage of a battery

$Q_{u}$ - Usable capacity of battery

For a generic $R C$ circuit pair, the current to voltage relationship can be written using (5) and (6).

$\frac{d V_{1}}{d t}=-\frac{V_{1}}{R_{1} C_{1}}+\frac{I_{b a t}}{C_{1}}$

$\frac{d V_{2}}{d t}=-\frac{V_{2}}{R_{2} C_{2}}+\frac{I_{b a t}}{C_{2}}$

In this model, $S O C, V_{1} \& V_{2}$ are considered as states for the state-space model, since these are not directly measured. $I_{b a t}$ is only one input variable, $V_{b a t}$ represents the model output.

\subsection{EKF algorithm:}

EKF is an appropriate choice for SOC estimation of battery because the battery is a nonlinear system. The EKF is a filter that estimates states based on statistical knowledge about states and noises (process noise and measurement noise) [28]. EKF uses basic matrix operations for state estimation, so it can be easily embedded for onboard BMS, making it useful in EV applications. The state estimation using EKF involves a prediction mode and a correction mode, 
as shown in Figure 3.

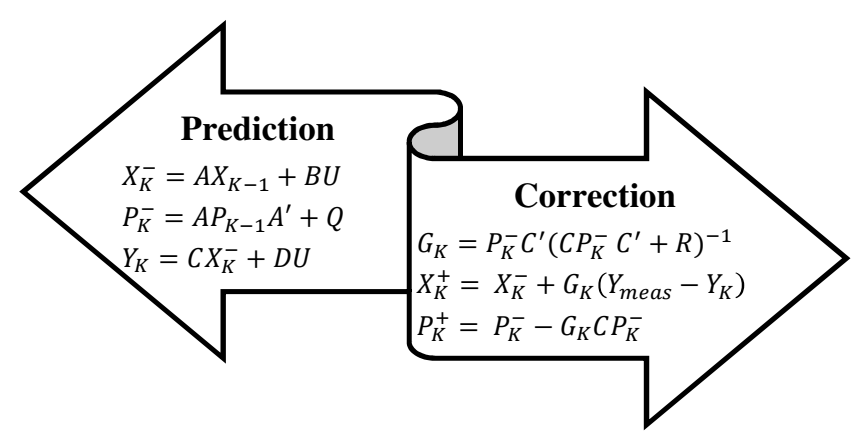

Figure 3 Calculation process of EKF

After initializing the state variables $\left(X_{K-1}\right)$ and error covariance matrix value $\left(P_{K-1}\right)$, predict the state matrix $\left(X_{K}^{-}\right)$and error covariance matrix $\left(P_{K}^{-}\right)$in the prediction mode. Then, Kalman gain $\left(G_{K}\right)$ is calculated to correct the state matrix $\left(X_{K}^{+}\right)$and error covariance matrix $\left(P_{K}^{+}\right)$ in the correction mode. $Y_{K}$ represents the predicted model output and $Y_{\text {meas }}$ is defined as the measured output.

EKF uses partial derivatives and first order Taylor series expansion to linearize the battery model. The state-space model is linearized at each time instance and the predicted value is compared with the measured terminal voltage of the battery to correct the state estimation. The estimated SOC is fed back into the battery model to update the model parameters for the next SOC estimation.

According to a set of aforementioned model equations, the discrete form of the state space equation of the two RC ECM is represented by (7) and (8).

$X_{K}=A X_{K-1}+B U$

$Y_{K}=C X_{K}+D U$ 
Since there are three state variables and one input variable, $A$ is a $3 \times 3$ matrix, $B$ is a $3 \times 1$ matrix, $C$ is a $1 \times 3$ matrix, $D$ is a $1 \times 1$ matrix. The values of $X, A, B, C, D$ matrices and the detailed EKF process are explained in Figure 4.

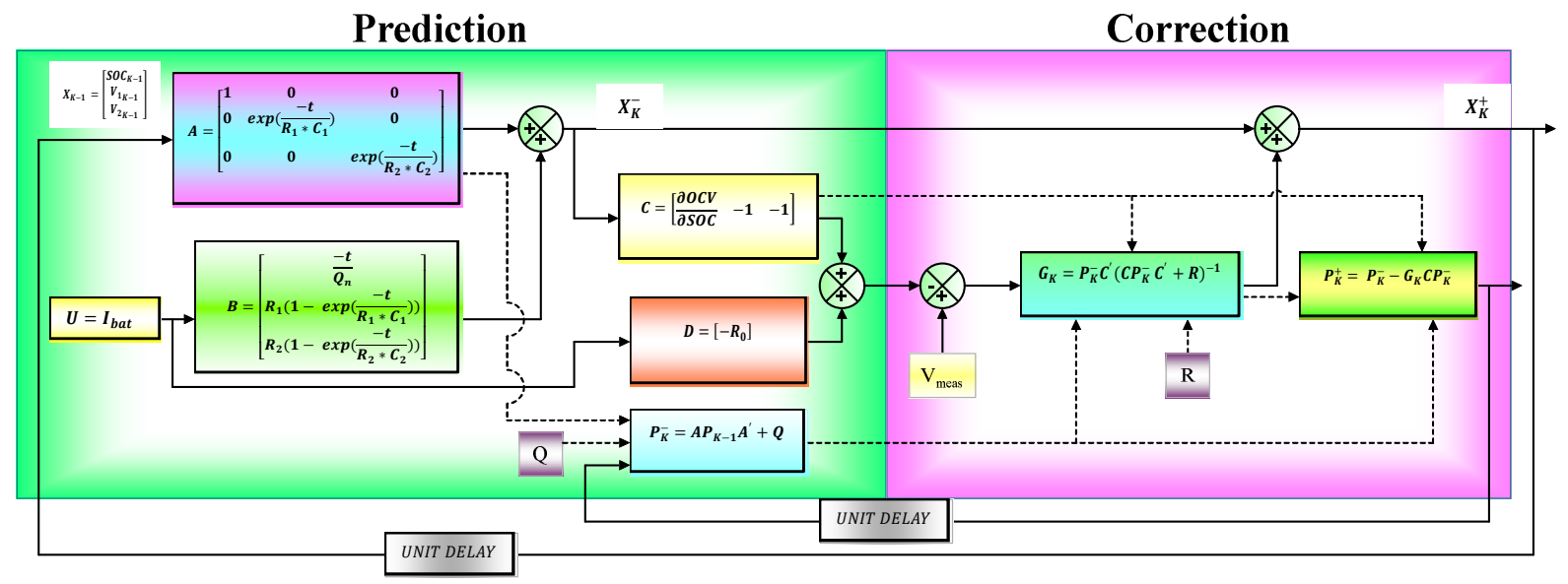

Figure 4 EKF framework with Two RC-ECM

Here, $U$ is measured current $\left(I_{\text {bat }}\right)$ and the same applies to the battery model to predict the state and output. The predicted output of the model is compared with the measured output $\left(V_{\text {meas }}\right)$. The sampling time is denoted by t. $\mathrm{P}$ is the error covariance matrix that is corrected at every time step. $Q \& R$ are the process and measurement covariance matrices of the Gaussian white noise vectors with the mean value of zero. During the first iteration, the state matrix and error covariance matrix are initialized. The corrected values of the state matrix and error covariance matrix are taken as an input for the prediction mode during the next iteration.

The values of the $\mathrm{Q} \& \mathrm{R}$ matrices have a significant impact on the EKF process's convergence rate and estimation error [53]. Picking a value for these matrices is a challenge because the noises are random and difficult to determine exactly. The impact of Q \& R matrices on estimation results is shown in Figure 5. Therefore, care must be taken to satisfy the overall performance of the EKF algorithm. 


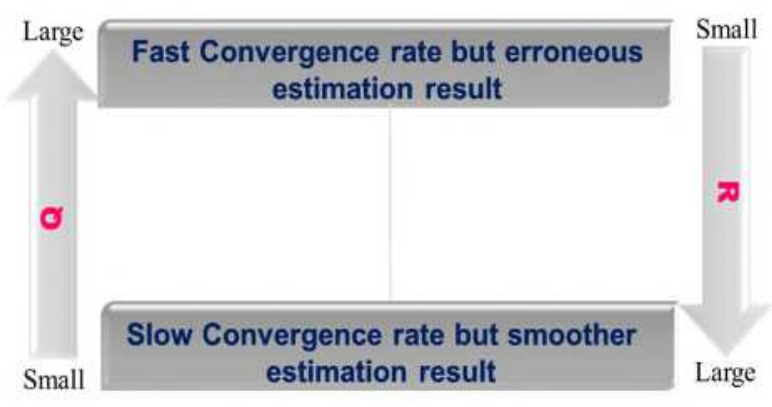

Figure 5 Impact of Q and R on estimation results

\subsection{Proposed SFO-EKF for SOC estimation:}

This paper introduces the SFO based EKF algorithm for SOC estimation. The SFO algorithm is introduced to find the optimal values of the noise covariance matrices ( $Q$ \& R), which play a vital role in EKF performance for accurate state estimation.

$\mathrm{SFO}$ is a metaheuristic, population-based algorithm inspired by nature behavior, described by G.F. Gomes in 2018 [54]. It requires only basic mathematical operators and is highly computationally efficient in terms of memory and speed. The SFO mimics the movement of sunflowers towards the sun, finding the global optimal solution professionally instead of getting stuck at the local optimal point. The main advantage of SFO is that it is derivative free when evaluating fitness function [55].

The main idea behind this SFO is to seek the best sunflower movement to absorb the radiation from the sun. The cycle of a sunflower is daily periodic. Every morning, sunflowers wake up and move towards the sun. In the evening, sunflowers go in the opposite direction, waiting for the next sunrise. The sunflower absorbs considerably more heat when it is so close to the sun, and it prefers to calm in this location. On the other hand, the distant sunflower receives less heat and takes more effort to reach as close to the global optimum as possible. The equation 9 describes how much heat each population receives. 
$Q_{i}=\frac{P}{4 \pi r_{i}^{2}}$

where, $Q_{i}$ is the heat received by $\mathrm{i}^{\text {th }}$ flower, $\mathrm{P}$ is the sun's power and $\mathrm{r}_{\mathrm{i}}$ is the distance between the sun and $\mathrm{i}^{\text {th }}$ flower.

Pollination in this algorithm is random through the minimum distance between flower $\mathrm{i}$ and flower $i+1$. Actually, a flower patch emits a huge amount of pollen gametes. To simplify the algorithm, it is assumed that each sunflower generates a single pollen gamete and is only copied once. The direction of the sunflowers towards the sun can be expressed in (10).

$\overrightarrow{s_{\imath}}=\frac{X^{*}-X_{i}}{\left\|X^{*}-X_{i}\right\|}, i=1,2, \ldots \ldots n$

The step of the sunflower in the sun direction is calculated according to the (11).

$d_{i}=\lambda \times P_{i}\left(|| X_{i}+X_{i+1}||\right) \times\left\|X_{i}+X_{i+1}\right\|$

where $\lambda$ is the constant value that defines an "inertial" displacement of plants, $P_{i}\left(|| X_{i}+X_{i+1}||\right)$ is the probability of pollination, i.e., the sunflower i pollinates its next neighbour to create a new individual in a random position. The new position is determined by the distance between the flowers.

Individuals closest to the sun will take smaller steps in pursuit of local refinement, while those far away will move regularly [56]. It is also vital to limit the maximum number of steps taken by each sunflower in order to avoid them violating the search space. The maximum step is defined by (12).

$d_{\max }=\frac{X_{\max }-X_{\min }}{2 \times N}$

where, $X_{\max }-X_{\min }$ is the difference between upper and lower bound values, and $\mathrm{N}$ is the number of flowers in the total population. The new plantation is considered by (13).

$\overrightarrow{X_{l+1}}=\overrightarrow{X_{l}}+d_{i} \times \overrightarrow{s_{l}}$ 
Figure 6 illustrates the steps of the SFO algorithm presented for parameter identification. The algorithm starts production of a population that can be random or even. The highest rated population of all is the one to be turned into the sun. Then these flowers orient themselves facing the sun and take random steps in a certain direction. The control parameters of SFO are pollination rate, mortality rate and survival rate. These parameters are chosen based on the condition such that the sum of these three parameters is unity [57].

With measured voltage and predicted voltage, the SFO tunes the Q \& R matrix values by calculating the fitness function for every sample of current. As per statistical knowledge, lower bounds and upper bounds are chosen for the Q \& R matrix. For each generation, the best values of the $\mathrm{Q} \& \mathrm{R}$ matrix are determined after evaluating the fitness function. After all generations are over, the optimal values of the Q \& R matrix that produce the minimum mean square error are obtained by SFO algorithm. In the proposed scheme, the SFO algorithm tunes the diagonal elements of matrices Q \& R offline, and these values are inserted into the corresponding matrices to estimate the SOC online. To obtain the optimal parameters, the EKF algorithm is executed for SOC estimation of a Li-ion battery which is discharged at a 1C-rate for one hour. 


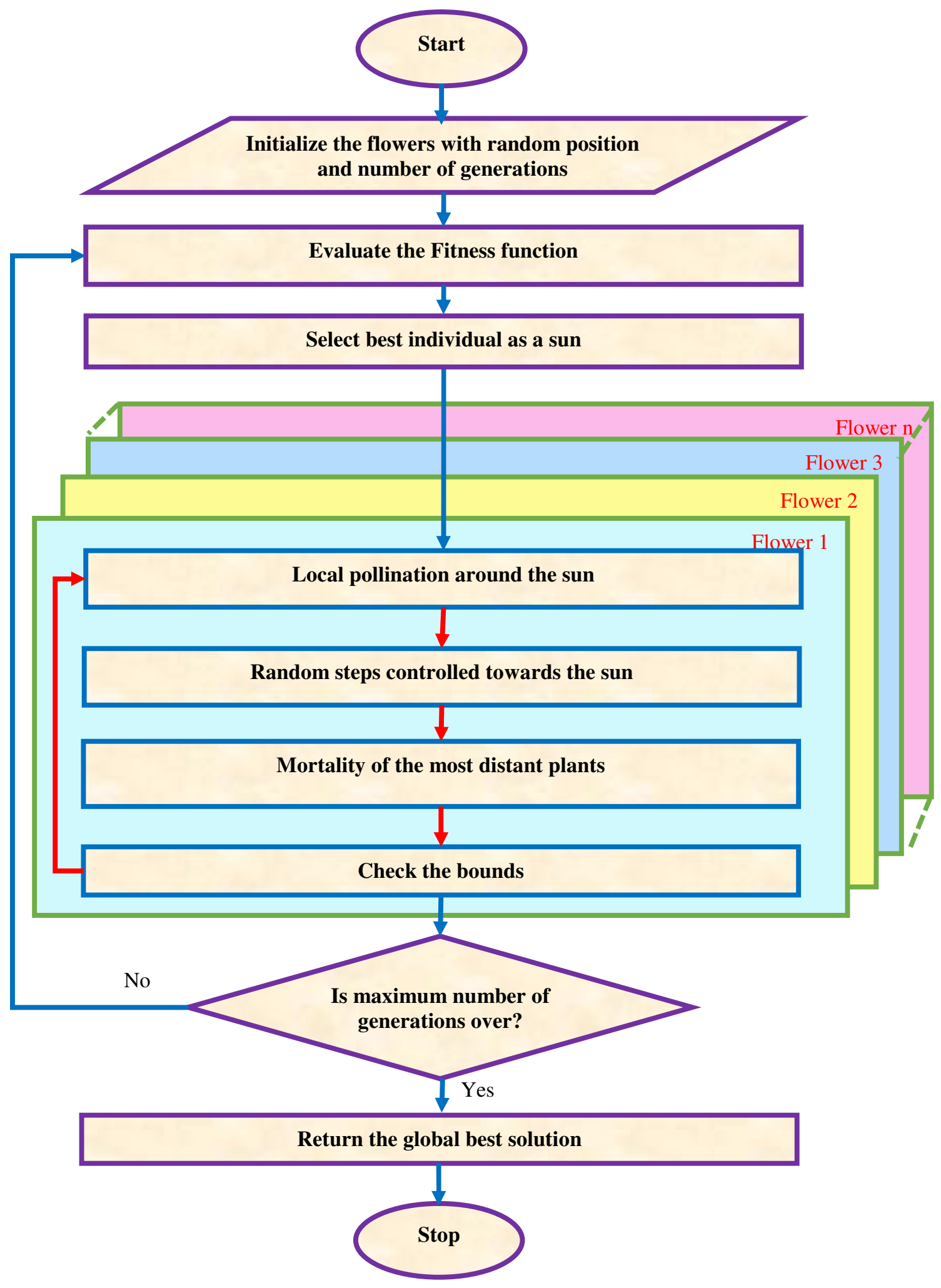

Figure 6 The entire process of SFO algorithm 


\section{SIMULATION RESULTS AND DISCUSSION}

The EKF estimation algorithm for two RC-ECM is verified and the block diagram is shown in Figure 4. The battery model is developed in Simulink and the EKF algorithm is coded in M-file. In a manufacturer's datasheet, charge and discharge currents are expressed in terms of a C-rate, where $1 \mathrm{C}$ is defined as the current needed to fully charge or discharge the nominal capacity of the battery in 1 hour.

For the discharging scenario, the initial SOC is assumed $100 \%$. So, state initial values are chosen as $\left[\begin{array}{lll}1 & 0 & 0\end{array}\right]^{\prime}$. Measured values are obtained from the manufacturer's catalog. Measured values are fed through the signal builder. The measured current is injected into the battery model. Since states are considered independent, the error covariance matrix is chosen as a diagonal matrix.

After each step, the corrected values are fed back into the system, i.e., the states and error covariance matrix are updated with a new value. In addition to this, A, B, C, D matrices are also updated since all parameters depend on SOC. Therefore, the time consumption of the parameter

identification process with the complex algorithm at each time step is reduced. The impact of noise covariance matrices on estimation is analyzed before the value is selected. For analysis purposes, only the Li-ion battery of the EEMB is used. Once appropriate values are chosen, the proposed ECM-based estimation algorithm is verified for discharging scenarios at various Crates. Li-ion discharge curves are obtained from two different manufacturers such as EEMB and SAFT datasheets.

\subsection{Impact of noise covariance matrices $Q$ and $R$ on estimation:}

$\mathrm{Q} \& \mathrm{R}$ values must be compromised between the estimation error and convergence rate in 
estimation. Incorrect values reduce the accuracy and make divergence in state estimation. When $\mathrm{Q}<<\mathrm{R}$, the estimated value slowly converges to measured data and gives a smooth result. Rising Q not only increases the convergence rate but also produces large estimation errors. On the other hand, Kalman gain is reduced when $\mathrm{R}$ is too large, which makes a diverging estimate. Tuning the values of $\mathrm{Q} \& \mathrm{R}$ is necessary for better estimation. For this analysis, simulation has been done for the constant current discharging of a Li-ion battery at a $1 \mathrm{C}$ rate. The effect of $\mathrm{Q} \& \mathrm{R}$ on voltage and SOC curves is shown in Figure 7 through 10 different datasets with measured values.

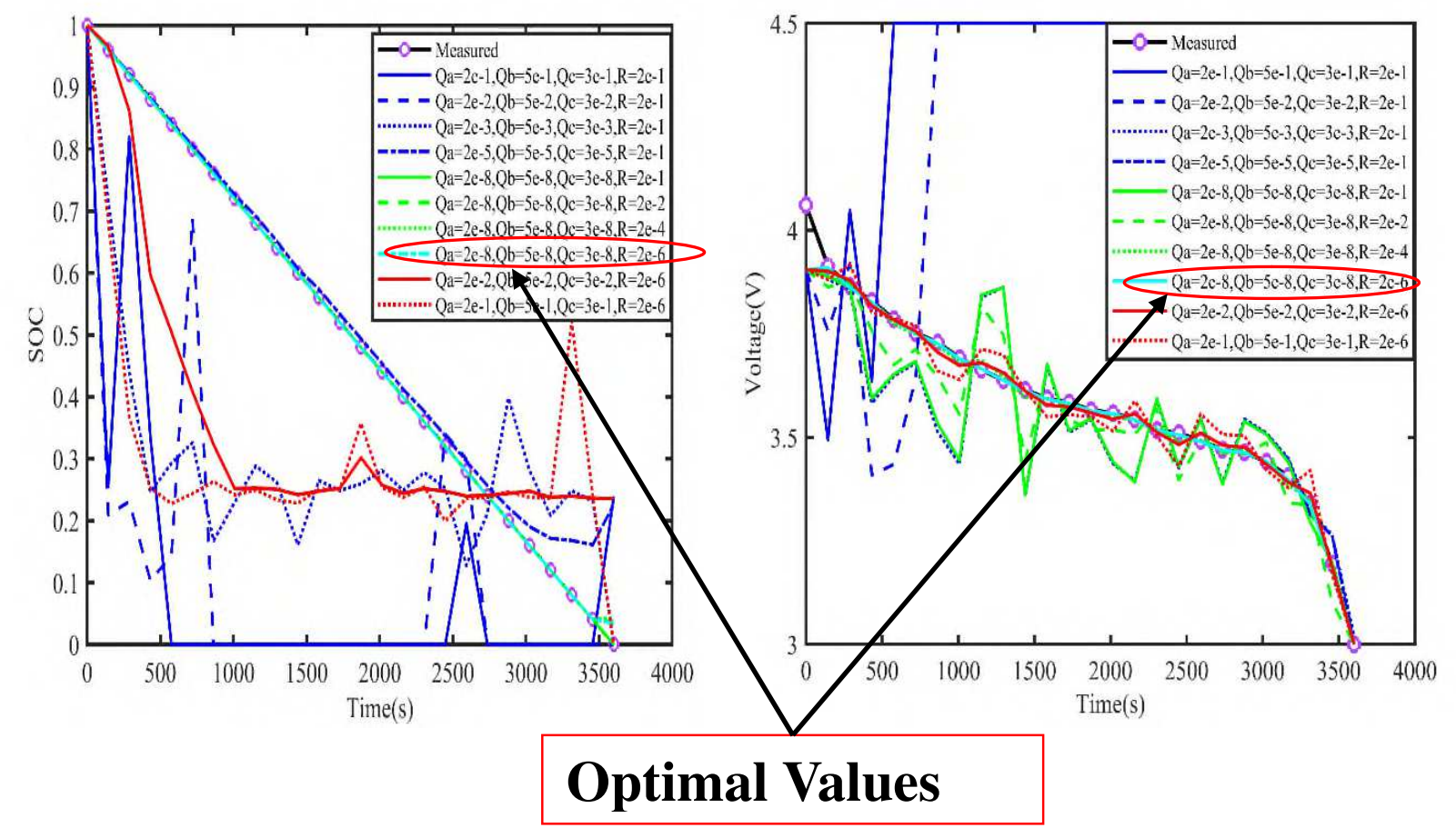

Figure 7 Selection of noise covariance matrices by comparing 10 different combinations with measured data

Since the simultaneous tuning of both matrices is very tedious, $Q$ matrix values are tuned while the $\mathrm{R}$ matrix is kept at a constant value. According to Figure 7, large Q-values increase the estimation error and the convergence rate. When $\mathrm{Q}$ is reduced, the state estimation error also decreases, resulting in smooth convergence, as shown in Figure 7. 
For the goodness of fit, mean square error (MSE) and root mean square error (RMSE) of SOC and voltage are calculated for different Q \& R values compared with measured data, which are shown in Table 1.

Table 1 Calculated MSE and RMSE for the goodness of fit

\begin{tabular}{|c|c|c|c|c|c|}
\hline \multirow{2}{*}{ S. No } & \multirow{2}{*}{$\begin{array}{l}\text { Diagonal elements of noise } \\
\text { covariance matrices }\end{array}$} & \multicolumn{2}{|c|}{ SOC } & \multicolumn{2}{|c|}{ Voltage (V) } \\
\hline & & MSE & RMSE & MSE & RMSE \\
\hline 1 & $\mathrm{Qa}=2 \mathrm{e}-1, \mathrm{Qb}=5 \mathrm{e}-1, \mathrm{Qc}=3 \mathrm{e}-1, \mathrm{R}=2 \mathrm{e}-1$ & 0.4127 & 0.6424 & 0.9840 & 0.9920 \\
\hline 2 & $\mathrm{Qa}=2 \mathrm{e}-2, \mathrm{Qb}=5 \mathrm{e}-2, \mathrm{Qc}=3 \mathrm{e}-2, \mathrm{R}=2 \mathrm{e}-1$ & 0.2363 & 0.4862 & 0.9300 & 0.9644 \\
\hline 3 & $\mathrm{Qa}=2 \mathrm{e}-3, \mathrm{Qb}=5 \mathrm{e}-3, \mathrm{Qc}=3 \mathrm{e}-3, \mathrm{R}=2 \mathrm{e}-1$ & 0.1041 & 0.3227 & 0.0797 & 0.2823 \\
\hline 4 & $\mathrm{Qa}=2 \mathrm{e}-5, \mathrm{Qb}=5 \mathrm{e}-3, \mathrm{Qc}=3 \mathrm{e}-3, \mathrm{R}=2 \mathrm{e}-1$ & 0.0033 & 0.0573 & 0.0800 & 0.2828 \\
\hline 5 & $\mathrm{Qa}=2 \mathrm{e}-8, \mathrm{Qb}=5 \mathrm{e}-3, \mathrm{Qc}=3 \mathrm{e}-3, \mathrm{R}=2 \mathrm{e}-1$ & 0.00004 & 0.0064 & 0.0797 & 0.2824 \\
\hline 6 & $\mathrm{Qa}=2 \mathrm{e}-8, \mathrm{Qb}=5 \mathrm{e}-3, \mathrm{Qc}=3 \mathrm{e}-3, \mathrm{R}=2 \mathrm{e}-2$ & 0.00005 & 0.0072 & 0.0222 & 0.1490 \\
\hline 7 & $\mathrm{Qa}=2 \mathrm{e}-8, \mathrm{Qb}=5 \mathrm{e}-3, \mathrm{Qc}=3 \mathrm{e}-3, \mathrm{R}=2 \mathrm{e}-4$ & 0.00005 & 0.0072 & 0.0014 & 0.0376 \\
\hline 8 & $\mathrm{Qa}=2 \mathrm{e}-8, \mathrm{Qb}=5 \mathrm{e}-3, \mathrm{Qc}=3 \mathrm{e}-3, \mathrm{R}=2 \mathrm{e}-6$ & 0.000001 & 0.0011 & 0.00022 & 0.0148 \\
\hline 9 & $\mathrm{Qa}=2 \mathrm{e}-2, \mathrm{Qb}=5 \mathrm{e}-2, \mathrm{Qc}=3 \mathrm{e}-2, \mathrm{R}=2 \mathrm{e}-6$ & 0.0909 & 0.3015 & 0.0011 & 0.0337 \\
\hline 10 & $\mathrm{Qa}=2 \mathrm{e}-1, \mathrm{Qb}=5 \mathrm{e}-1, \mathrm{Qc}=3 \mathrm{e}-1, \mathrm{R}=2 \mathrm{e}-6$ & 0.2976 & 0.5455 & 0.0028 & 0.0529 \\
\hline
\end{tabular}

After a tuning by trial \& error method, process noise and measurement noise covariance matrices are selected as follows:

$$
\begin{gathered}
Q=\left[\begin{array}{ccc}
2 e^{-8} & 0 & 0 \\
0 & 5 e^{-3} & 0 \\
0 & 0 & 3 e^{-3}
\end{array}\right] \\
R=\left[2 e^{-6}\right]
\end{gathered}
$$

\subsection{Tuning of noise covariance matrices by SFO algorithm:}

Noise covariance matrices must be properly tuned to ensure accurate SOC estimation. Optimality of the EKF must be ensured by an optimization algorithm in order to tune the $\mathrm{Q} \& \mathrm{R}$ matrices. For SOC estimation of EV batteries, EKF, which is optimized by SFO, is presented for SOC estimation. The SFO algorithm is a nature-inspired metaheuristic algorithm which is applied to 
tune the diagonal elements of $\mathrm{Q} \& \mathrm{R}$ matrices by evaluating the fitness function. To obtain the unknown parameters of EKF, such as the process noise covariance matrix and measurement noise covariance matrix, a fitness function can be defined as the difference between the calculated values of the SOC and the estimated values obtained from the EKF. The minimization of this function allows the SFO to find the best values of EKF parameters. After defining the fitness function, SFO parameters are initialized before the execution, as shown in Table 2. The stopping criteria is set as the maximum number of generations. When stopping criteria are met, optimum values of matrices $\mathrm{Q}$ and $\mathrm{R}$ are obtained.

\begin{tabular}{|c|c|c|c|}
\hline \multicolumn{2}{|l|}{ SFO } & \multicolumn{2}{|l|}{ GA } \\
\hline Parameter & Value & Parameter & Value \\
\hline Number of sunflowers & 40 & Population & 40 \\
\hline Pollination rate & 0.1 & Crossover & 0.8 \\
\hline Mortality rate & 0.1 & Mutation & 0.1 \\
\hline Survival rate & 0.8 & - & - \\
\hline Number of generations & 100 & Number of generations & 100 \\
\hline Sun & 1 & Elitism & 1 \\
\hline
\end{tabular}

For performance evaluation of the proposed SFO-EKF, an EKF which is designed by the trial \& error method (TR-EKF), GA-EKF, and AEKF are used for baseline comparison. Figure 8 illustrates the comparison of four approaches which are used for SOC estimation of electric vehicle batteries. It can be noticed that the SOC estimation using SFO-EKF is close to the results obtained from the AEKF method. The accuracy of SFO is much more noticeable in the zoomed area when compared to the other methods. The comparison of numerical results obtained by four methods is shown in Table 3. Figures 9a and $9 \mathrm{~b}$ depict the SOC and voltage error profile respectively. 


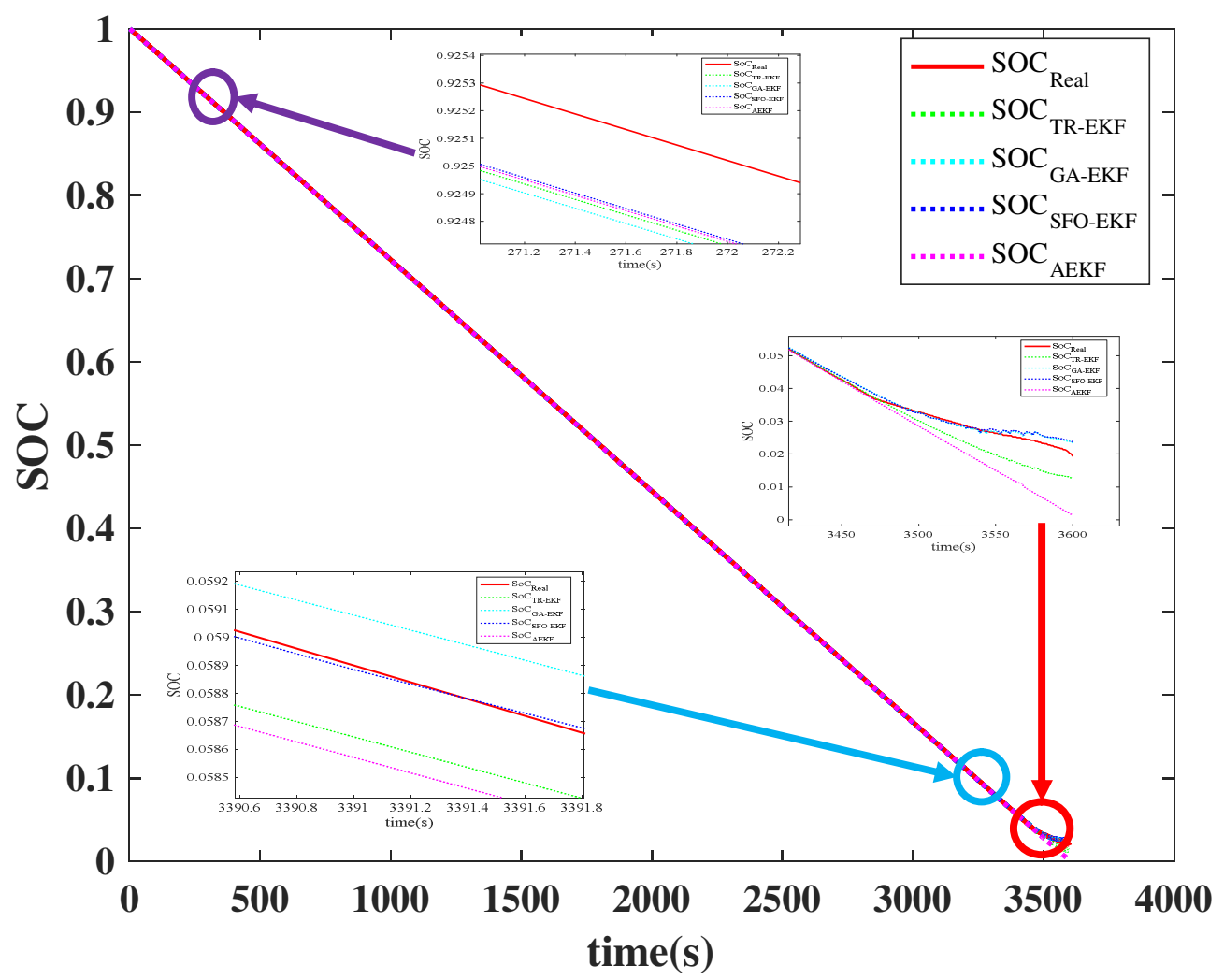

Figure 8 Comparison of results obtained from four approaches used for SOC estimation

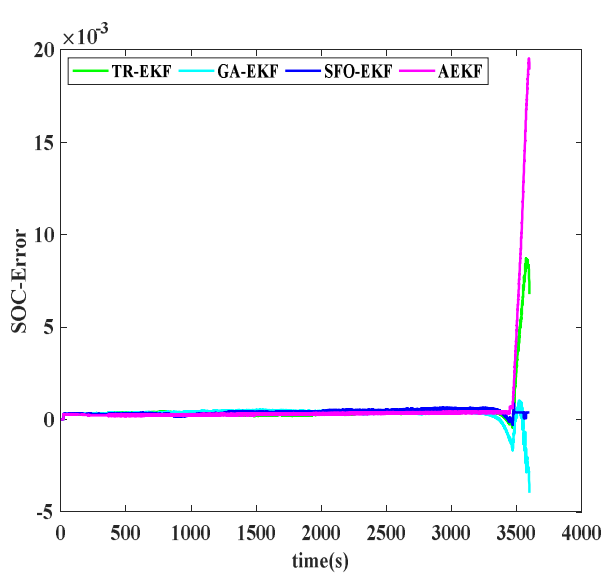

Figure 9a Comparison of SOC error profile obtained from four methods used for SOC estimation

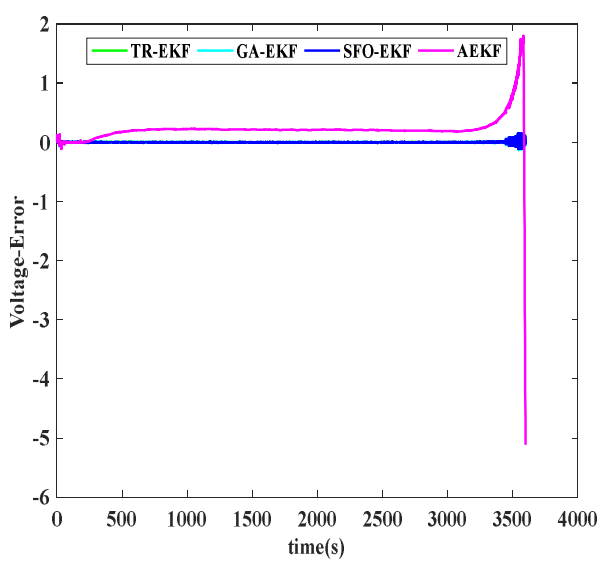

Figure 9b Comparison of Voltage error profile obtained from four methods used for SOC estimation 
Table 3 Comparison of numerical results obtained by TR-EKF, GA-EKF, SFO-EKF and AEKF

\begin{tabular}{|c|cccc||cccc|}
\hline \multirow{2}{*}{ METHOD } & \multicolumn{5}{|c||}{ SOC } & \multicolumn{4}{c|}{ VOLTAGE(V) } \\
\cline { 2 - 8 } & Max. Error & MAE & MSE & RMSE & Max. Error & MAE & MSE & RMSE \\
\hline TR-EKF & 0.0087 & $4.9444 \mathrm{e}-04$ & $1.328 \mathrm{e}-06$ & 0.0011 & 0.1594 & 0.0052 & $2.1919 \mathrm{e}-04$ & 0.0148 \\
& & & & & & & & \\
GA-EKF & 0.0010 & $4.0734 \mathrm{e}-04$ & $2.279 \mathrm{e}-07$ & $4.774 \mathrm{e}-04$ & 0.1528 & 0.0051 & $2.0562 \mathrm{e}-04$ & 0.01433 \\
& & & & & & & & \\
SFO-EKF & $\mathbf{6 . 1 8 2 e - 0 4}$ & $\mathbf{1 . 0 1 3 e - 0 4}$ & $\mathbf{3 . 1 5 8 e - 0 8}$ & $\mathbf{1 . 7 7 7 e - 0 4}$ & $\mathbf{0 . 1 4 9 9}$ & $\mathbf{0 . 0 0 5 0}$ & $\mathbf{2 . 0 3 0 8 e - 0 4}$ & $\mathbf{0 . 0 1 4 2 5}$ \\
& & & & & & & & \\
AEKF & 0.0009 & $4.032 \mathrm{e}-04$ & $2.392 \mathrm{e}-07$ & $4.891 \mathrm{e}-04$ & 0.8970 & 0.0067 & 0.0033 & 0.0581 \\
\hline
\end{tabular}

As observed, the performance indices such as maximum error, Mean Absolute Error (MAE), MSE and RMSE of both SOC and voltage obtained by the proposed SFO-EKF are low compared to the other three methods. The maximum SOC error produced by SFO-EKF is 6.182e-04, which is lower compared with the other three methods. Furthermore, the RMSE of SFO-EKF is lowered by $83.8 \%, 62.8 \%$ and $63.7 \%$ compared with TR-EKF, GA-EKF and AEKF respectively. As a result, it is possible to conclude that the proposed method can enhance estimation accuracy when compared to the other methods.

Aside from estimation accuracy, convergence speed is a significant performance metric. Figure 10 depicts the SOC estimation results at a $20 \%$ initial SOC inaccuracy. In this example, the proposed method and AEKF require less than 50 seconds to complete the convergence, whereas GA-EKF and TR-EKF require 70 seconds and 90 seconds, respectively, as shown in the zoomed area of Figure 10. It also reveals that the deviation of the initial SOC has a low impact on estimation accuracy. 


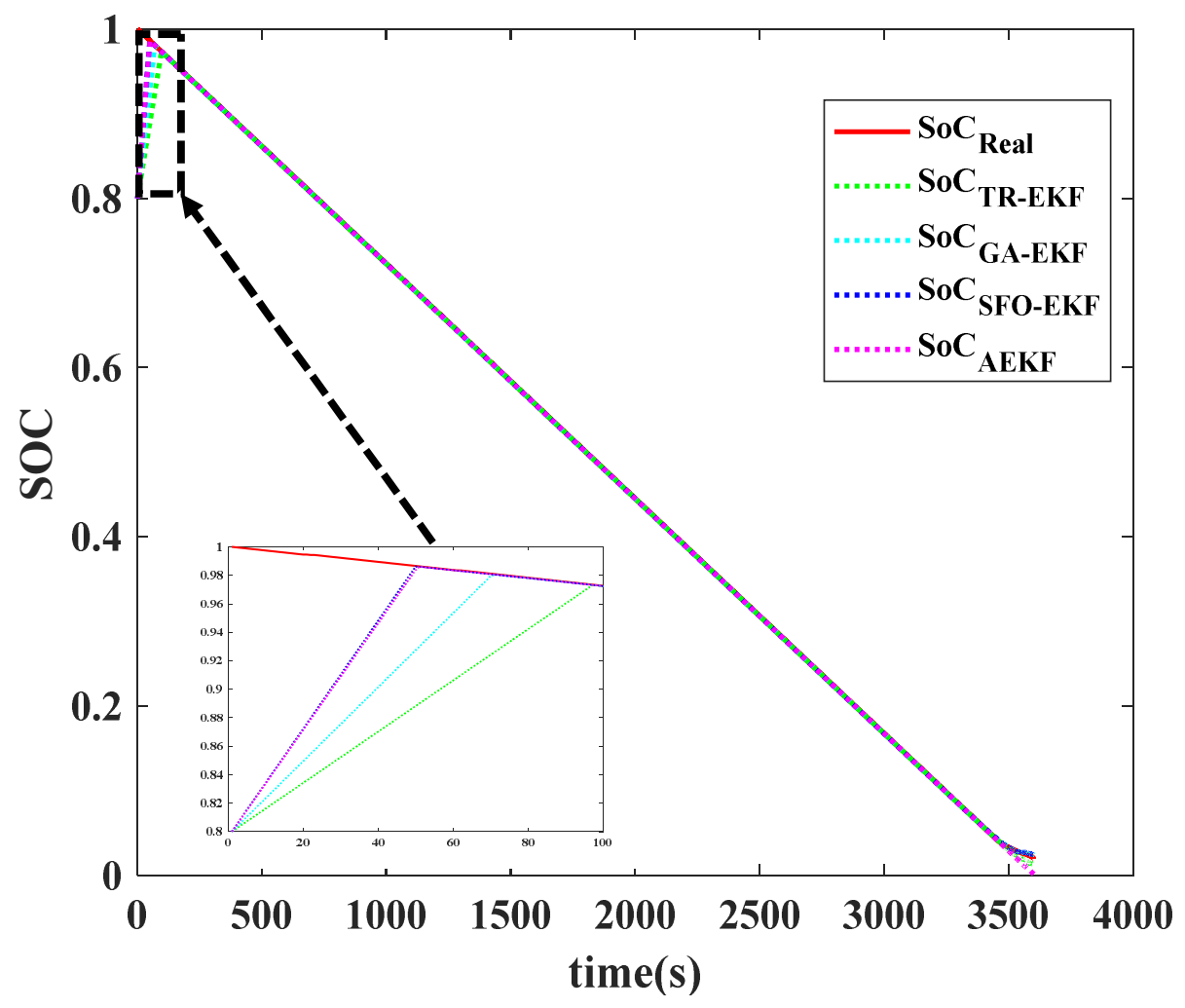

Figure 10 SOC estimation results at a $20 \%$ erroneous initial SOC

As per the above discussion, it is worth mentioning that the SFO-EKF method outperforms the AEKF, GA-EKF, and TR-EKF algorithms in terms of estimation accuracy and convergence speed.

\subsection{SFO-EKF validation:}

The test situations in this study are divided into constant current and dynamic current situations in order to completely validate the effectiveness of the proposed method. The constant current discharging test replicates the operation of an EV when the power battery is discharged repeatedly at an almost constant current under constant speed conditions. The dynamic current condition is used to charge and discharge the cell at pre-set and changing current rates to simulate the operating conditions in the actual driving process. In this paper, two battery 
manufacturers' datasheets (EEMB, SAFT) and an online dataset from NASA are used for the constant current discharging test. Meanwhile, the Dynamic Stress Test (DST) cycle is a common dynamic driving cycle that is widely used to assess and analyse the SOC estimation of lithiumion batteries.

The SFO-EKF algorithm is verified with two battery manufacturer's data for constant current discharging setups at various C-rates. For comparison, measured data is represented as a solid line, where a marker represents the result obtained via the EKF algorithm in each figure. Then, the effect of the C-rate on the complete discharge characteristic of the battery is discussed.

\subsubsection{EEMB battery}

The battery's nominal capacity is listed as $2600 \mathrm{mAh}$ on the specification sheet, so 1C corresponds to $2.6 \mathrm{~A}$ current. Figure 11 a shows the battery fully discharged in one hour with $2.6 \mathrm{~A}$ constant current. The discharge voltage and SOC with respect to time are shown in Figure 11a. It is noticed that the end of discharge voltage is $3 \mathrm{~V}$, which matches the datasheet.




Figure 11b shows that the battery is fully discharged in five hours (18000 seconds). Figure $11 \mathrm{~b}$ shows the battery that is discharged from $4.15 \mathrm{~V}$ to $3.5 \mathrm{~V}$ in five hours. It is noticed that the end of discharge voltage is above the discharge cut-off voltage. So, this is the standard and safe discharge current for the battery as mentioned in the spec sheet.

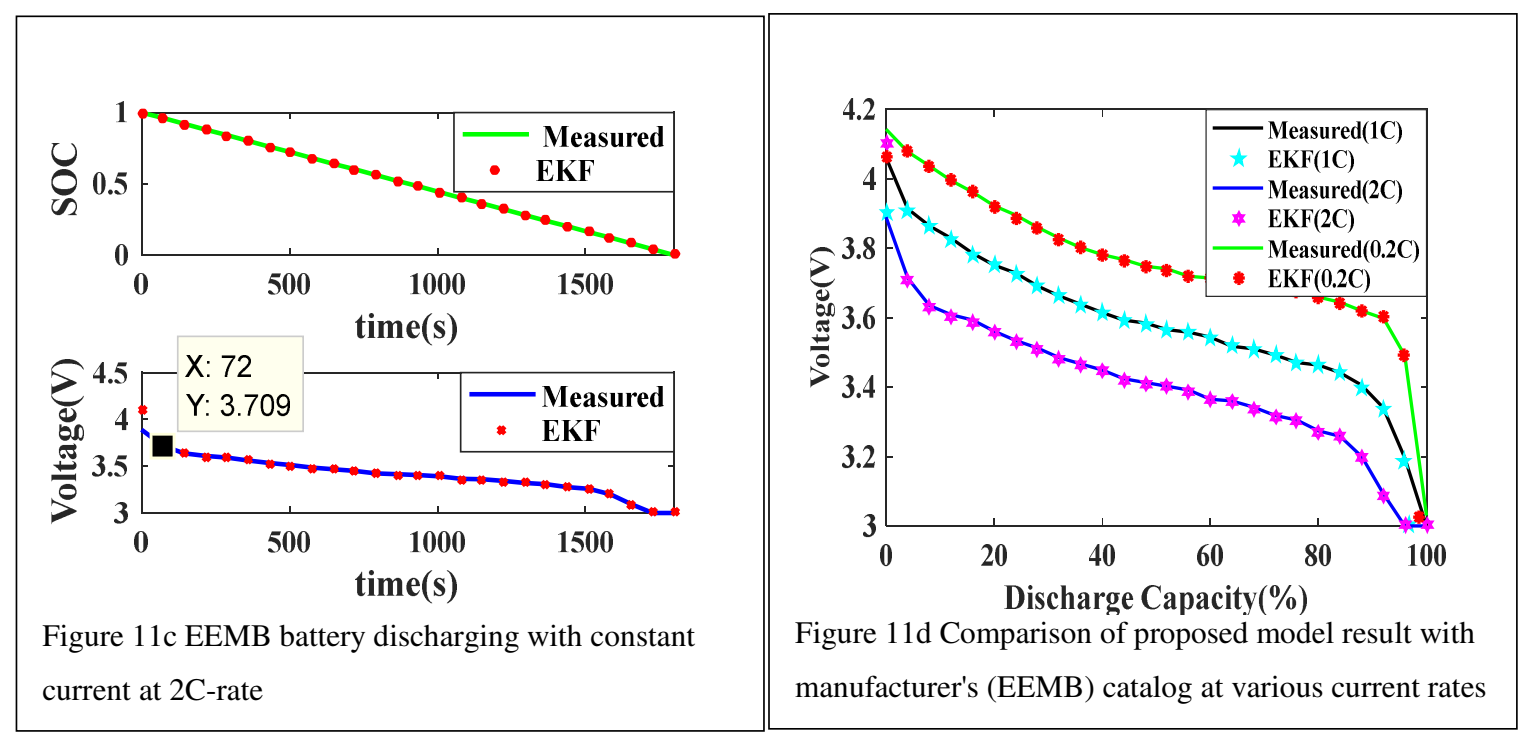

According to the datasheet, the average value of the battery's working voltage, i.e., nominal voltage is $3.7 \mathrm{~V}$. But Figure $11 \mathrm{c}$ shows that the battery discharge voltage at Time $=72 \mathrm{~s}$ is 3.7V. Therefore, the discharge current rate of more than $1 \mathrm{C}$ is not advisable for optimal battery utilization.

According to Figure 11d, the percentage of the battery's discharge capacity becomes lower when the discharge current rate is increased. In general, battery discharging efficiency is less than charging efficiency. In addition, an increase in the $\mathrm{C}$ rate has an extreme effect on the efficiency of battery discharge. 


\subsubsection{SAFT battery}

Here, the battery nominal capacity is $6.8 \mathrm{Ah}$ and the discharge cut-off voltage is $2.5 \mathrm{~V}$. Battery discharging curves at various constant current rates such as $1 \mathrm{C}(6.8 \mathrm{~A}), 0.2 \mathrm{C}(1.36 \mathrm{~A})$ and 2C (13.6A) and compared with measured data are shown in Figure 12a, Figure 12b and Figure $12 \mathrm{c}$ respectively.

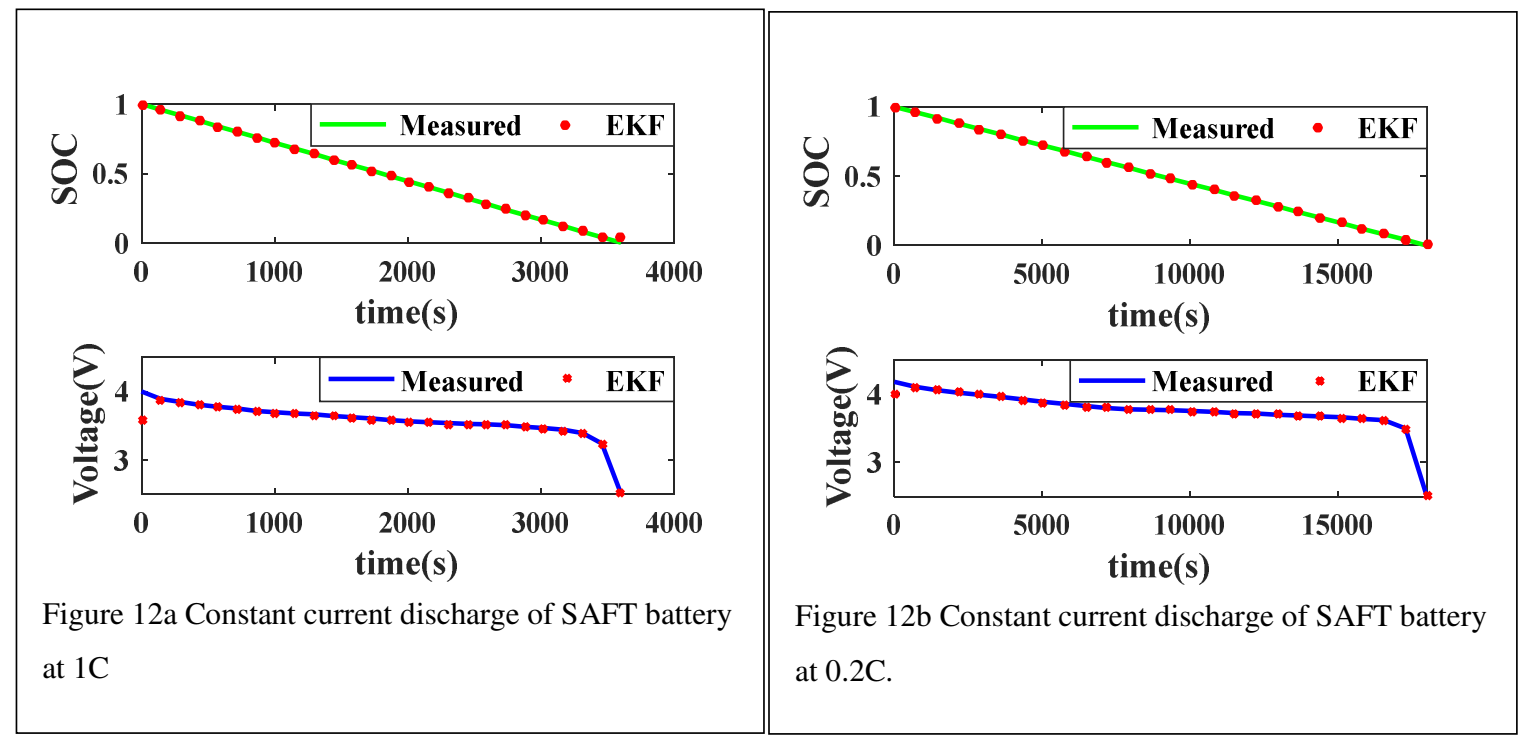

It is noticed that the proposed algorithm closely yields discharging curves of the battery manufacturers. The nominal voltage of a battery is $3.75 \mathrm{~V}$. When the current drawn from the battery is more than $6.8 \mathrm{~A}$, the battery rapidly decreases from $4.2 \mathrm{~V}$ to $3.75 \mathrm{~V}$ (nominal voltage) within a minute. Therefore, the battery is working well when the discharge current rate is less than 1C-rate. Figure 12d shows the complete discharge characteristics, i.e., Discharge capacity (\%) vs Voltage (V) for three different C-rates.

This article performs simulation work at three different $\mathrm{C}$ rates $(1 \mathrm{C}, 2 \mathrm{C}$, and $0.2 \mathrm{C})$ for the discharge scenario, and compares the corresponding results with the measured value from the datasheet. For three different C-rates, the proposed model result compared with curves obtained from the manufacturer's datasheet such as EEMB and SAFT to verify the complete discharge 
properties (discharge capacity vs. discharge voltage) of the battery (see Figure 11d and Figure $12 d)$.

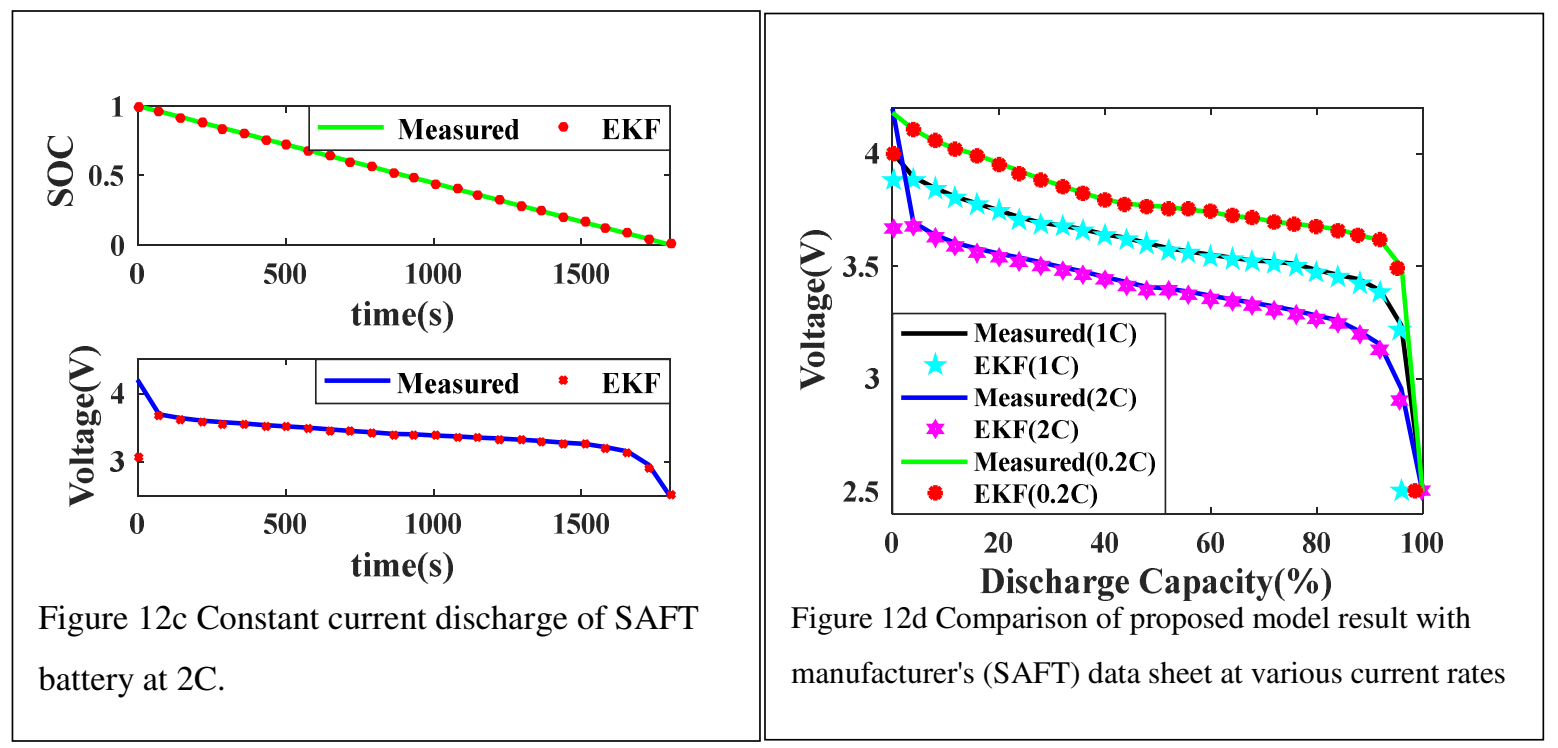

Based on the results, it is noticed that the discharge characteristic curves of the developed algorithm match very well with the curves from the manufacturer's datasheet. In addition, the discharge capacity decreases when the C-rate increases. Therefore, a low C-rate is recommended to extend battery life. The SFO-EKF gives acceptable performance when the discharge rate changes.

\subsubsection{Online dataset from NASA}

In addition to the datasheet, an online dataset from NASA [58] have been used to verify the effectiveness of the proposed algorithm. Measured current and voltage of Battery 5 in the NASA dataset are given to the algorithm and SOC is calculated using the Ah method, which is used as a reference SOC. The results obtained from SFO-EKF are superimposed with the curve obtained from the NASA dataset, as shown in Figure 13. 

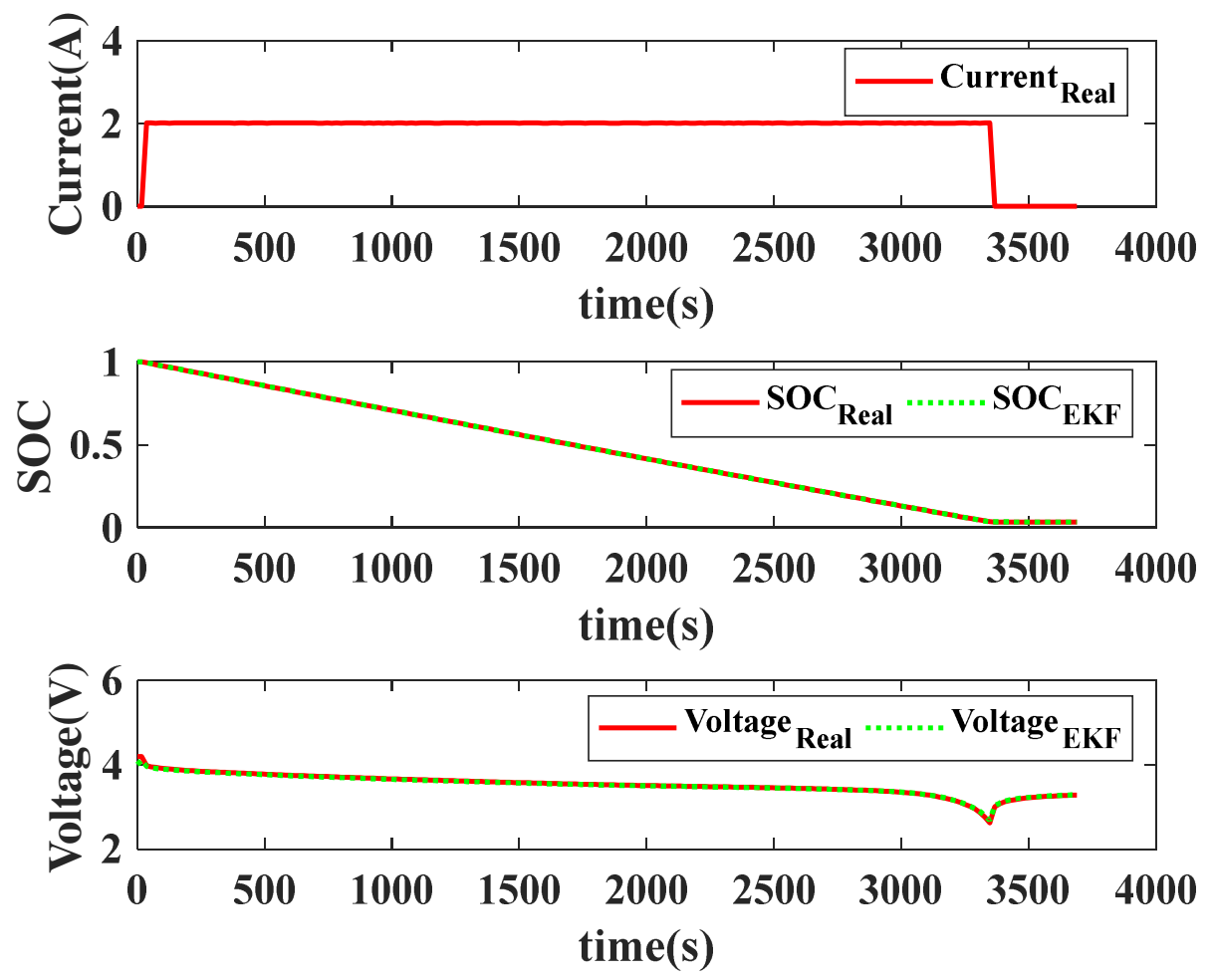

Figure 13 SFO-EKF algorithm validation on SOC estimation using the NASA dataset

\subsubsection{Dynamic Stress Test (DST) profile}

To better simulate the loading behavior of the EV battery in real time, DST profile is used. The DST data for the A123-18650 Li-ion battery, which has a battery capacity of 1.1Ah and a nominal voltage of $3.7 \mathrm{~V}$, is taken from [59]. The current profile, as shown in Figure 14, contains a variety of current amplitudes. The proposed method is validated by measuring the battery current and voltage during DST for one cycle (360 seconds). Figure 14 indicates that the SOC computed using the proposed method is identical to the SOC obtained using the Ampere hour technique for the DST driving cycle. As a result, the validity of the SFO-EKF is confirmed under varying loads. Since the Q \& R matrices have been optimized with respect to the constant discharge at $1 \mathrm{C}$-rate, the performance of the SFO-EKF is satisfactory for various operating conditions of an EV battery. 

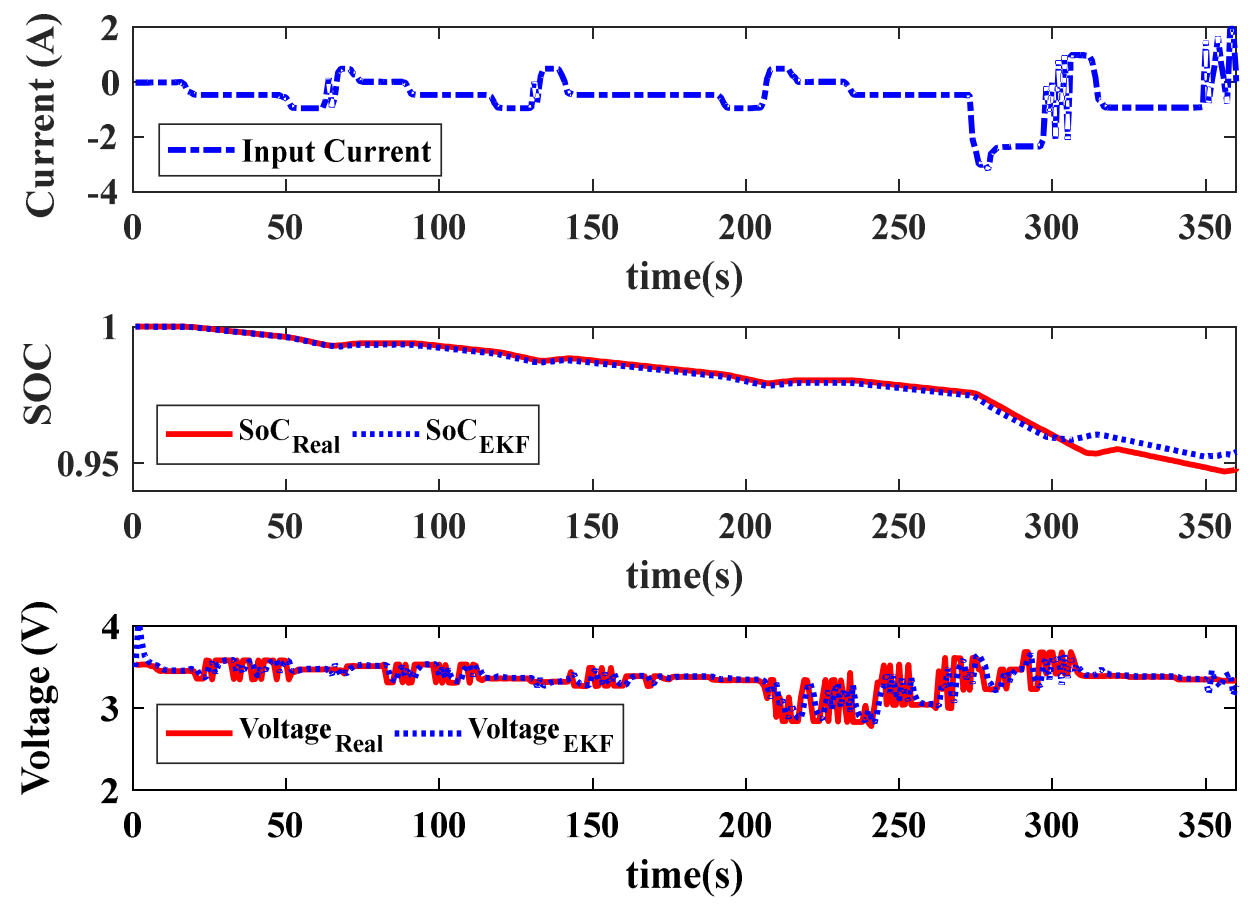

Figure 14 SFO-EKF algorithm validation on SOC estimation under DST

\section{CONCLUSION}

The process noise covariance matrix $\mathrm{Q}$ and measurement noise covariance matrix $\mathrm{R}$ have an impact on the EKF state estimation process. Incorrect noise covariance matrices reduce state estimation accuracy and cause divergence. In this paper, the effect of noise covariance matrices (Q \& R) on state estimation has been analyzed using the trial \& error method. Next, the Sunflower optimization algorithm (SFO) is proposed as a method for choosing the optimum Q \& $\mathrm{R}$ values. The proposed SFO-EKF method is compared to the Adaptive Extended Kalman filter, GA-EKF, and EKF tuning using the trial \& error method in terms of accuracy and convergence rate. The maximum SOC error produced by SFO-EKF is lower compared with the other three 
methods. Furthermore, the RMSE of SFO-EKF is lowered by $83.8 \%, 62.8 \%$ and $63.7 \%$ compared with EKF tuning by trial \& error method, GA-EKF and Adaptive Extended Kalman filter, respectively. In addition, the proposed method converged quickly with the reference SOC when the erroneous initial SOC is $20 \%$. Based on the numerical results, it is possible to conclude that the proposed method can enhance estimation accuracy and convergence speed when compared to other methods. The battery discharge characteristics are analyzed with various current rates for two different battery chemistries. In addition, the proposed algorithm is verified with the online dataset from NASA and the dynamic stress test profile of an electric vehicle. Based on the comparative results, it can be concluded that the proposed SFO-EKF for SOC estimation is an effective technique for the prediction process of a dynamic system.

\section{ACKNOWLEDGMENT}

The authors gratefully acknowledge Alagappa Chettiar Government College of Engineering and Technology, Karaikudi for the support under TEQIP.

\section{CONFLICTS OF INTEREST / COMPETING INTERESTS}

The authors have no conflicts of interest to declare that are relevant to the content of this article.

\section{DATA AVAILABILITY STATEMENT}

The battery datasets analysed during the current study are freely available in the NASA Ames Prognostics Data Repository, NASA Ames Research Center, Moffett Field, CA, 2007. [https://ti.arc.nasa.gov/c/5/].

This dataset was downloaded from the following public Data Repository:

[http://ti.arc.nasa.gov/project/prognostic-data-repository] 


\section{REFERENCES}

[1] S. Juyal et al., "ZERO EMISSION VEHICLES (ZEVs): TOWARDS A POLICY FRAMEWORK,” 2018. [Online]. Available: http://niti.gov.in/writereaddata/files/document_publication/EV_report.pdf

[2] Z. Zhang, L. Zhang, L. Hu, and C. Huang, "Active cell balancing of lithium-ion battery pack based on average state of charge,” International Journal of Energy Research, vol. 44, no. 4, pp. 2535-2548, 2020, doi: 10.1002/er.4876.

[3] A. Farjah, T. Ghanbari, and A. R. Seifi, "Contribution management of lead-acid battery, Li-ion battery, and supercapacitor to handle different functions in EVs," International Transactions on Electrical Energy Systems, vol. 30, no. 1, pp. 1-21, 2020, doi: 10.1002/2050-7038.12155.

[4] A. Dinger et al., "Batteries for Electric Cars Challenges, Opportunities, and the Outlook to 2020," 2020. [Online]. Available: www.bcg.com/publications

[5] H. Chaoui, C. C. Ibe-Ekeocha, and H. Gualous, "Aging prediction and state of charge estimation of a LiFePO4 battery using input time-delayed neural networks," Electric Power Systems Research, vol. 146, pp. 189-197, May 2017, doi: 10.1016/j.epsr.2017.01.032.

[6] Maheshwari Adaikkappan and Nageswari Sathiyamoorthy, "Modeling, state of charge estimation, and charging of lithium-ion battery in electric vehicle: A review," International Journal of Energy Research, no. April, pp. 1-25, 2021, doi: 10.1002/er.7339.

[7] R. Xiong, J. Cao, Q. Yu, H. He, and F. Sun, "Critical Review on the Battery State of Charge Estimation Methods for Electric Vehicles," IEEE Access, vol. 6, pp. 1832-1843, Dec. 2017, doi: 10.1109/ACCESS.2017.2780258.

[8] Z. Xu, S. Gao, and S. Yang, "LiFePO4 battery state of charge estimation based on the improved Thevenin equivalent circuit model and Kalman filtering," Journal of Renewable and Sustainable Energy, vol. 8, no. 2, 2016, doi: $10.1063 / 1.4944335$.

[9] K. Liu, K. Li, Q. Peng, and C. Zhang, "A brief review on key technologies in the battery management system of electric vehicles,” Frontiers of Mechanical Engineering, vol. 14, no. 1. Higher Education Press, pp. 47-64, Mar. 01, 2019. doi: $10.1007 / \mathrm{s} 11465-018-0516-8$.

[10] J. Xie, J. Ma, and K. Bai, "Enhanced coulomb counting method for state-of-charge estimation of lithium-ion batteries based on peukert's law and coulombic efficiency," Journal of Power Electronics, vol. 18, no. 3, pp. 910-922, 2018, doi: 10.6113/JPE.2018.18.3.910.

[11] J. Meng et al., "An Overview and Comparison of Online Implementable SOC Estimation Methods for Lithium-Ion Battery,” IEEE Transactions on Industry Applications, vol. 54, no. 2, 2018, doi: 10.1109/TIA.2017.2775179.

[12] X. Dang, L. Yan, K. Xu, X. Wu, H. Jiang, and H. Sun, "Open-Circuit Voltage-Based State of Charge Estimation of Lithium-ion Battery Using Dual Neural Network Fusion Battery Model,” Electrochimica Acta, vol. 188, pp. 356-366, 2016, doi: 10.1016/j.electacta.2015.12.001.

[13] Y. Xing, W. He, M. Pecht, and K. L. Tsui, "State of charge estimation of lithium-ion batteries using the open-circuit voltage at various ambient temperatures," Applied Energy, vol. 113, pp. 106-115, 2014, doi: 10.1016/j.apenergy.2013.07.008.

[14] M. U. Ali, A. Zafar, S. H. Nengroo, S. Hussain, M. J. Alvi, and H. J. Kim, "Towards a smarter battery management system for electric vehicle applications: A critical review of lithium-ion battery state of charge estimation," Energies, vol. 12, no. 3. MDPI AG, Jan. 30, 2019. doi: 10.3390/en12030446. 
[15] R. Zhang et al., "State of the art of lithium-ion battery SOC estimation for electrical vehicles," Energies, vol. 11 , no. 7. MDPI AG, 2018. doi: 10.3390/en11071820.

[16] T. O. Ting, K. L. Man, N. Zhang, C. U. Lei, and C. Lu, "State-space battery modeling for smart Battery Management System,” Lecture Notes in Engineering and Computer Science, vol. 2210, no. January, pp. 12-15, 2014.

[17] C. Zhang, K. Li, S. McLoone, and Z. Yang, "Battery modelling methods for electric vehicles - A review," in 2014 European Control Conference, ECC 2014, Jul. 2014, pp. 2673-2678. doi: 10.1109/ECC.2014.6862541.

[18] R. Zhang et al., "State of the art of lithium-ion battery SOC estimation for electrical vehicles," Energies, vol. 11 , no. 7. MDPI AG, pp. 1-36, 2018. doi: 10.3390/en11071820.

[19] W. Allafi, K. Uddin, C. Zhang, R. Mazuir Raja Ahsan Sha, and J. Marco, "On-line scheme for parameter estimation of nonlinear lithium ion battery equivalent circuit models using the simplified refined instrumental variable method for a modified Wiener continuous-time model," Applied Energy, vol. 204, pp. 497-508, 2017, doi: 10.1016/j.apenergy.2017.07.030.

[20] D. N. T. How, M. A. Hannan, M. S. Hossain Lipu, and P. J. Ker, "State of Charge Estimation for Lithium-Ion Batteries Using Model-Based and Data-Driven Methods: A Review,” IEEE Access, vol. 7, pp. 136116-136136, 2019, doi: 10.1109/ACCESS.2019.2942213.

[21] V. M. Nagulapati et al., "A novel combined multi-battery dataset based approach for enhanced prediction accuracy of data driven prognostic models in capacity estimation of lithium ion batteries," Energy and AI, vol. 5, p. 100089, 2021, doi: 10.1016/j.egyai.2021.100089.

[22] C. Zhang, Y. Zhu, G. Dong, and J. Wei, "Data-driven lithium-ion battery states estimation using neural networks and particle filtering," International Journal of Energy Research, vol. 43, no. 14, pp. 8230-8241, Nov. 2019, doi: 10.1002/ER.4820.

[23] J. Du, Z. Liu, and Y. Wang, "State of charge estimation for Li-ion battery based on model from extreme learning machine," Control Engineering Practice, vol. 26, no. 1, pp. 11-19, 2014, doi: 10.1016/j.conengprac.2013.12.014.

[24] C. Zhang, W. Allafi, Q. Dinh, P. Ascencio, and J. Marco, "Online estimation of battery equivalent circuit model parameters and state of charge using decoupled least squares technique," Energy, vol. 142, pp. 678-688, Jan. 2018, doi: 10.1016/j.energy.2017.10.043.

[25] H. Yang, X. Sun, Y. An, X. Zhang, T. Wei, and Y. Ma, "Online parameters identification and state of charge estimation for lithium-ion capacitor based on improved Cubature Kalman filter," Journal of Energy Storage, vol. 24, no. March, p. 100810, 2019, doi: 10.1016/j.est.2019.100810.

[26] F. Asghar, M. Talha, S. H. Kim, and I. H. Ra, "Simulation study on battery state of charge estimation using Kalman Filter," Journal of Advanced Computational Intelligence and Intelligent Informatics, vol. 20, no. 6, pp. 861-866, 2016, doi: 10.20965/jaciii.2016.p0861.

[27] U. Syamkumar and B. Jayanand, "Real-time implementation of sensorless indirect field-oriented control of three-phase induction motor using a Kalman smoothing-based observer," International Transactions on Electrical Energy Systems, vol. 30, no. 2, pp. 1-17, 2020, doi: 10.1002/2050-7038.12242.

[28] Y. Laamari, K. Chafaa, and B. Athamena, "Particle swarm optimization of an extended Kalman filter for speed and rotor flux estimation of an induction motor drive," Electrical Engineering, vol. 97, no. 2, pp. 129-138, 2015, doi: 10.1007/s00202-014-0322-1.

[29] P. Shrivastava, T. Kok Soon, M. Y. I. bin Idris, S. Mekhilef, and S. B. R. S. Adnan, "Combined State of Charge and State of Energy Estimation of Lithium-Ion Battery Using Dual Forgetting Factor-Based Adaptive Extended Kalman 
Filter for Electric Vehicle Applications," IEEE Transactions on Vehicular Technology, vol. 70, no. 2, pp. 1200-1215, Feb. 2021, doi: 10.1109/TVT.2021.3051655.

[30] Z. He, Z. Yang, X. Cui, and E. Li, “A Method of State-of-Charge Estimation for EV Power Lithium-Ion Battery Using a Novel Adaptive Extended Kalman Filter," IEEE Transactions on Vehicular Technology, vol. 69, no. 12, pp. 1461814630, Dec. 2020, doi: 10.1109/TVT.2020.3032201.

[31] J. Meng, G. Luo, and F. Gao, "Lithium polymer battery state-of-charge estimation based on adaptive unscented kalman filter and support vector machine," IEEE Transactions on Power Electronics, 2016, doi: 10.1109/TPEL.2015.2439578.

[32] S. Zhang, X. Guo, and X. Zhang, "An improved adaptive unscented kalman filtering for state of charge online estimation of lithium-ion battery," Journal of Energy Storage, vol. 32, no. October, p. 101980, 2020, doi: 10.1016/j.est.2020.101980.

[33] G. Liu, C. Xu, K. Jiang, and K. Wang, "State of charge and model parameters estimation of liquid metal batteries based on adaptive unscented Kalman filter," Energy Procedia, vol. 158, pp. 4477-4482, 2019, doi: 10.1016/j.egypro.2019.01.765.

[34] N. Peng, S. Zhang, X. Guo, and X. Zhang, "Online parameters identification and state of charge estimation for lithiumion batteries using improved adaptive dual unscented Kalman filter," International Journal of Energy Research, vol. 45, no. 1, pp. 975-990, Jan. 2021, doi: 10.1002/ER.6088.

[35] S. Peng, C. Chen, H. Shi, and Z. Yao, "State of charge estimation of battery energy storage systems based on adaptive unscented Kalman filter with a noise statistics estimator," IEEE Access, vol. 5, pp. 13202-13212, 2017, doi: 10.1109/ACCESS.2017.2725301.

[36] X. Shu, G. Li, J. Shen, Z. Lei, Z. Chen, and Y. Liu, “An adaptive multi-state estimation algorithm for lithium-ion batteries incorporating temperature compensation," Energy, vol. 207, p. 118262, 2020, doi: 10.1016/j.energy.2020.118262.

[37] P. Shrivastava, T. K. Soon, M. Y. I. bin Idris, and S. Mekhilef, "Overview of model-based online state-of-charge estimation using Kalman filter family for lithium-ion batteries," Renewable and Sustainable Energy Reviews, vol. 113, no. June, p. 109233, 2019, doi: 10.1016/j.rser.2019.06.040.

[38] C. Campestrini, T. Heil, S. Kosch, and A. Jossen, "A comparative study and review of different Kalman filters by applying an enhanced validation method," Journal of Energy Storage, vol. 8, pp. 142-159, 2016, doi: 10.1016/j.est.2016.10.004.

[39] T. O. Ting, K. L. Man, E. G. Lim, and M. Leach, “Tuning of Kalman Filter Parameters via Genetic Algorithm for Stateof-Charge Estimation in Battery Management System,” The Scientific World Journal, vol. 2014, pp. 1-11, 2014, doi: $10.1155 / 2014 / 176052$.

[40] K. L. Shi, T. F. Chan, Y. K. Wong, and S. L. Ho, "Speed estimation of an induction motor drive using an optimized extended Kalman filter," IEEE Transactions on Industrial Electronics, vol. 49, no. 1, pp. 124-133, 2002, doi: 10.1109/41.982256.

[41] Jianguo Yan, Dongli Yuan, Xiaojun Xing, and Qiuling Jia, "Kalman filtering parameter optimization techniques based on genetic algorithm," in Proceedings of the IEEE International Conference on Automation and Logistics Qingdao, China, 2008, no. September, pp. 1717-1720. doi: 10.1109/ical.2008.4636432.

[42] S. Mishra, S. Ghosh, and A. Goswami, "A genetic algorithm tuned kalman filter for estimating harmonic and interharmonic attributes," 2017 4th International Conference on Power, Control and Embedded Systems, ICPCES 2017, vol. 2017-Janua, pp. 1-4, 2017, doi: 10.1109/ICPCES.2017.8117652. 
[43] O. Tremblay, L.-A. Dessaint, and A.-I. Dekkiche, "A Generic Battery Model for the Dynamic Simulation of Hybrid Electric Vehicles," in IEEE International vehicle power propulsion conference, 2007, pp. 284-289.

[44] F. Kazhamiaka, S. Keshav, C. Rosenberg, and K. H. Pettinger, "Simple spec-based modeling of lithium-ion batteries," IEEE Transactions on Energy Conversion, vol. 33, no. 4, pp. 1757-1765, Dec. 2018, doi: 10.1109/TEC.2018.2838441.

[45] K. Thirugnanam, T. P. Ezhil Reena Joy, M. Singh, and P. Kumar, "Mathematical modeling of li-ion battery using genetic algorithm approach for V2G applications," IEEE Transactions on Energy Conversion, vol. 29, no. 2, pp. 332343, 2014, doi: 10.1109/TEC.2014.2298460.

[46] A. Sakti et al., "Enhanced representations of lithium-ion batteries in power systems models and their effect on the valuation of energy arbitrage applications," Journal of Power Sources, vol. 342, pp. 279-291, 2017, doi: 10.1016/j.jpowsour.2016.12.063.

[47] A. Malik, Z. Zhang, and R. K. Agarwal, "Extraction of battery parameters using a multi-objective genetic algorithm with a non-linear circuit model," Journal of Power Sources, vol. 259, pp. 76-86, Aug. 2014, doi: 10.1016/j.jpowsour.2014.02.062.

[48] M. Chen and G. A. Rincón-Mora, "Accurate electrical battery model capable of predicting runtime and I-V performance," IEEE Transactions on Energy Conversion, vol. 21, no. 2, pp. 504-511, Jun. 2006, doi: 10.1109/TEC.2006.874229.

[49] C. Paleologu, J. Benesty, and S. Ciochina, "A practical variable forgetting factor recursive least-squares algorithm," 2014 11th International Symposium on Electronics and Telecommunications, ISETC 2014 - Conference Proceedings, no. 3, pp. 3-6, 2015, doi: 10.1109/ISETC.2014.7010812.

[50] F. Albu, "Improved variable forgetting factor recursive least square algorithm," 2012 12th International Conference on Control, Automation, Robotics and Vision, ICARCV 2012, vol. 2012, no. December, pp. 1789-1793, 2012, doi: 10.1109/ICARCV.2012.6485421.

[51] T. Ouyang, P. Xu, J. Chen, J. Lu, and N. Chen, "Improved parameters identification and state of charge estimation for lithium-ion battery with real-time optimal forgetting factor," Electrochimica Acta, vol. 353, 2020, doi: 10.1016/j.electacta.2020.136576.

[52] X. Qiu, W. Wu, and S. Wang, "Remaining useful life prediction of lithium-ion battery based on improved cuckoo search particle filter and a novel state of charge estimation method," Journal of Power Sources, vol. 450, no. January, pp. 1-13, Feb. 2020, doi: 10.1016/j.jpowsour.2020.227700.

[53] W. Wang and J. Mu, "State of Charge Estimation for Lithium-Ion Battery in Electric Vehicle Based on Kalman Filter Considering Model Error,” IEEE Access, vol. 7, pp. 29223-29235, 2019, doi: 10.1109/ACCESS.2019.2895377.

[54] G. F. Gomes, S. S. da Cunha, and A. C. Ancelotti, "A sunflower optimization (SFO) algorithm applied to damage identification on laminated composite plates," Engineering with Computers, vol. 35, no. 2, pp. 619-626, 2018, doi: $10.1007 / \mathrm{s} 00366-018-0620-8$.

[55] M. A. M. Shaheen, H. M. Hasanien, S. F. Mekhamer, and H. E. A. Talaat, "Optimal power flow of power systems including distributed generation units using sunflower optimization algorithm," IEEE Access, vol. 7, pp. 109289109300, 2019, doi: 10.1109/ACCESS.2019.2933489.

[56] A. M. Hussien, H. M. Hasanien, and S. F. Mekhamer, "Sunflower optimization algorithm-based optimal PI control for enhancing the performance of an autonomous operation of a microgrid," Ain Shams Engineering Journal, vol. 12, pp. 1883-1893, 2021, doi: 10.1016/j.asej.2020.10.020. 
[57] G. F. Gomes and F. A. de Almeida, "Tuning metaheuristic algorithms using mixture design: Application of sunflower optimization for structural damage identification," Advances in Engineering Software, vol. 149, no. June 2019, 2020, doi: 10.1016/j.advengsoft.2020.102877.

[58] B. Saha and K. Goebel, "Battery Data Set," NASA Ames Prognostics Data Repository, NASA Ames Research Center, Moffett Field, CA, 2007. http://ti.arc.nasa.gov/project/prognostic-data-repository (accessed Aug. 07, 2021).

[59] F. Yang, S. Zhang, W. Li, and Q. Miao, "State-of-charge estimation of lithium-ion batteries using LSTM and UKF," Energy, vol. 201, p. 117664, 2020, doi: 10.1016/j.energy.2020.117664. 